\title{
Nonlocal Variational Principles with Variable Growth
}

\author{
Yongqiang Fu and Miaomiao Yang \\ Department of Mathematics, Harbin Institute of Technology, Harbin 150001, China \\ Correspondence should be addressed to Miaomiao Yang; 3d07@163.com
}

Received 17 January 2014; Accepted 6 March 2014; Published 7 April 2014

Academic Editor: Qingying Bu

Copyright (C) 2014 Y. Fu and M. Yang. This is an open access article distributed under the Creative Commons Attribution License, which permits unrestricted use, distribution, and reproduction in any medium, provided the original work is properly cited.

This paper is concerned with the functional $J$ defined by $J(u)=\int_{\Omega \times \Omega} W(x, y, \nabla u(x), \nabla u(y)) d x d y$, where $\Omega \subset \mathbb{R}^{N}$ is a regular open bounded set and $W$ is a real-valued function with variable growth. After discussing the theory of Young measures in variable exponent Sobolev spaces, we study the weak lower semicontinuity and relaxation of $J$.

\section{Introduction}

In this paper, we are concerned with a type of variational integrals that can be written as

$$
J(u)=\int_{\Omega \times \Omega} W(x, y, \nabla u(x), \nabla u(y)) d x d y
$$

where $\Omega \subset \mathbb{R}^{N}$ is a regular open bounded set $(N \geq 1)$, $u \in W^{1, p(x)}\left(\Omega ; \mathbb{R}^{m}\right)$, and $p(x)$ is Lipschitz continuous with $1<p_{-}:=\inf _{x \in \bar{\Omega}} p(x) \leq p(x) \leq p_{+}:=\sup _{x \in \bar{\Omega}} p(x)<\infty . W=$ $W\left(x, y, \lambda_{1}, \lambda_{2}\right): \mathbb{R}^{N} \times \mathbb{R}^{N} \times \mathbb{M}^{m \times N} \times \mathbb{M}^{m \times N} \rightarrow \mathbb{R}$ satisfies that $x \mapsto W\left(x, y, \lambda_{1}, \lambda_{2}\right)$ and $y \mapsto W\left(x, y, \lambda_{1}, \lambda_{2}\right)$ are measurable and $\lambda_{1} \mapsto W\left(x, y, \lambda_{1}, \lambda_{2}\right)$ and $\lambda_{2} \mapsto W\left(x, y, \lambda_{1}, \lambda_{2}\right)$ are continuous. $W$ is also assumed to satisfy the variable growth condition

$$
\begin{aligned}
c\left(\left|\lambda_{1}\right|^{p(x)}+\left|\lambda_{2}\right|^{p(x)}-1\right) & \leq W\left(x, y, \lambda_{1}, \lambda_{2}\right) \\
& \leq C\left(\left|\lambda_{1}\right|^{p(x)}+\left|\lambda_{2}\right|^{p(x)}+1\right),
\end{aligned}
$$

for $0<c<C$. Notice that

$$
\begin{gathered}
L^{p(x)}\left(\Omega, \mathbb{R}^{m}\right)=\left\{u: \Omega \subset \mathbb{R}^{N} \longrightarrow \mathbb{R}^{m},|u| \in L^{p(x)}(\Omega, \mathbb{R})\right\}, \\
W^{1, p(x)}\left(\Omega, \mathbb{R}^{m}\right)=\left\{u: \Omega \subset \mathbb{R}^{N} \longrightarrow \mathbb{R}^{m}, u \in L^{p(x)}\left(\Omega, \mathbb{R}^{m}\right),\right. \\
\left.\nabla u \in L^{p(x)}\left(\Omega, \mathbb{M}^{m \times N}\right)\right\} .
\end{gathered}
$$

After Kováčik and Rákosník first discussed $L^{p(x)}(\Omega)$ and $W^{m, p(x)}(\Omega)$ spaces in [1], a lot of research has been done concerning these kinds of variable exponent spaces; for examples, see [2-6] for the properties of such spaces and [7-9] for the applications of variable exponent spaces on partial differential equations. These problems with variable exponent growth possess very complicated nonlinearities; for instance, the $p(x)$-Laplacian operator is inhomogeneous. In recent years, these problems have received considerable attention and raised many difficult mathematical problems. The theory on various mathematical problems with $p(x)$ growth conditions has important applications in nonlinear elastic mechanics, imaging processing, electrorheological fluids. and other physics phenomena (see $[10,11]$ ).

The main goal of this paper is to study the weak lower semicontinuity and relaxation of the nonlocal variational problems. We will analyze these problems in terms of Young measures generated by sequences in variable exponent space. When $p(x)$ is a constant function, Pedregal studied problem (1) in [12]. In connection with variational problems of nonlocal nature the reader can consult [13] for problems related to ferromagnetism, [14] about the regularization of a nonconvex problem, and [15-17] in order to analyze mechanical problems formulated in general context of nonlocal elasticity. In [18] some interesting tools to obtain a full relaxation of specific nonlocal variational problems have been analyzed, and [19] is also a remarkable work for a general class of nonlocal integral functionals. If convexity condition does not hold for $W$, then we would like to have an equivalent variational principle in the sense that the infimum 
is preserved and the relaxed variational principle admits a minimizer. The usual way to proceed for local functionals is to replace $W$ by its convex hull. In nonlocal setting, there is no substitute for the convex hull. The only possible way to describe relaxation for these nonlocal variational principles is by using Young measures and a generalized functional defined on them (see $[12,20])$.

This paper is organized as follows. In Section 2, several important properties on variable exponent spaces are recalled; in Section 3, we give some conclusions of Young measures in variable exponent spaces; in Section 4, we analyze the weak lower semicontinuity and relaxation of problem (1).

\section{Preliminaries}

In this section, we first recall some facts on variable exponent spaces $L^{p(x)}(\Omega)$ and $W^{k, p(x)}(\Omega)$. See $[1,4-6]$ for the details.

Let $\mathbf{P}(\Omega)$ be the set of all Lebesgue measurable functions $p: \Omega \rightarrow[1,+\infty]$, where $\Omega \subset \mathbb{R}^{n}(n \geq 2)$ is a nonempty open subset. Denote

$$
\begin{gathered}
\rho_{p(x)}(u)=\int_{\Omega \backslash \Omega_{\infty}}|u(x)|^{p(x)} d x+\sup _{\Omega_{\infty}}|u(x)|, \\
\|u\|_{p(x)}=\inf \left\{t>0: \rho_{p(x)}\left(\frac{u}{t}\right) \leq 1\right\},
\end{gathered}
$$

where $\Omega_{\infty}=\{x \in \Omega: p(x)=\infty\}$. The variable exponent Lebesgue space $L^{p(x)}(\Omega)$ is the class of all functions $u$ such that $\rho_{p(x)}\left(t_{0} u\right)<\infty$ for some $t_{0}>0 . L^{p(x)}(\Omega)$ is a Banach space endowed with the norm (5). Equation (4) is called the modular of $u$ in $L^{p(x)}(\Omega)$.

For a given $p(x) \in \mathbf{P}(\Omega)$, we define the conjugate function $p^{\prime}(x)$ as

$$
p^{\prime}(x)= \begin{cases}\infty, & \text { if } x \in \Omega_{1}=\{x \in \Omega: p(x)=1\}, \\ 1, & \text { if } x \in \Omega_{\infty}, \\ \frac{p(x)}{p(x)-1}, & \text { for other } x \in \Omega .\end{cases}
$$

Lemma 1 (see [6]). Let $p \in \mathbf{P}(\Omega)$, then the inequality

$$
\int_{\Omega}|u(x) v(x)| d x \leq 2\|u\|_{p(x)}\|v\|_{p^{\prime}(x)}
$$

holds for every $u \in L^{p(x)}(\Omega), v \in L^{p^{\prime}(x)}(\Omega)$.

In the following parts of this section, for every $p \in \mathbf{P}(\Omega)$, we assume $1 \leq p_{-} \leq p(x) \leq p_{+}<\infty$.

Lemma 2 (see [5]). For any $u \in L^{p(x)}(\Omega)$, we have

(1) if $\|u\|_{p(x)} \geq 1$, then $\|u\|_{p(x)}^{p_{-}} \leq \rho_{p(x)}(u) \leq\|u\|_{p(x)}^{p_{+}}$;

(2) if $\|u\|_{p(x)}<1$, then $\|u\|_{p(x)}^{p_{+}} \leq \rho_{p(x)}(u) \leq\|u\|_{p(x)}^{p_{-}}$.

Lemma 3 (see [5]). If $p_{-}>1, L^{p(x)}(\Omega)$ is reflexive, and the dual space of $L^{p(x)}(\Omega)$ is $L^{p^{\prime}(x)}(\Omega)$.
Lemma 4 (see $[1]$ ). Let $|\Omega|<\infty$, where $|\Omega|$ denotes the Lebesgue measure of $\Omega$, and $p_{1}(x), p_{2}(x) \in \mathbf{P}(\Omega)$; then the necessary and sufficient condition for $L^{p_{2}(x)}(\Omega) \subset L^{p_{1}(x)}(\Omega)$ is that $p_{1}(x) \leq p_{2}(x)$ for almost every $x \in \Omega$, and in this case the embedding is continuous.

Next $k$ is a given positive integer. Given a multi-index $\alpha=\left(\alpha_{1}, \ldots, \alpha_{n}\right) \in \mathbb{N}^{n}$, we set $|\alpha|=\alpha_{1}+\cdots+\alpha_{n}$ and $D^{\alpha}=$ $D_{1}^{\alpha_{1}} \cdots D_{n}^{\alpha_{n}}$, where $D_{i}=\partial / \partial x_{i}$ is the generalized derivative operator.

The variable exponent Sobolev space $W^{k, p(x)}(\Omega)$ is the class of functions $u$ on $\Omega$ such that $D^{\alpha} u \in L^{p(x)}(\Omega)$ for every multi-index $\alpha$ with $|\alpha| \leq k \cdot W^{k, p(x)}(\Omega)$ is a Banach space endowed with the norm

$$
\|u\|_{k, p(x)}=\sum_{|\alpha| \leq k}\left\|D^{\alpha} u\right\|_{p(x)} .
$$

By $W_{0}^{k, p(x)}(\Omega)$ we denote the subspace of $W^{k, p(x)}(\Omega)$ which is the closure of $C_{0}^{\infty}(\Omega)$ with respect to the norm (8).

For any $u \in W^{1, p(x)}(\Omega)$, define

$$
\||u|\|=\inf \left\{t>0: \int_{\Omega} \frac{|\nabla u|^{p(x)}+|u|^{p(x)}}{t^{p(x)}} d x \leq 1\right\} .
$$

Then $\||\cdot| \mid$ is an equivalent norm of $W^{1, p(x)}(\Omega)$. If $\Omega$ is a bounded domain, $\|\nabla u\|_{p(x)}$ is an equivalent norm of $W_{0}^{1, p(x)}(\Omega)$.

Lemma 5 (see [1]). The spaces $W^{k, p(x)}(\Omega)$ and $W_{0}^{k, p(x)}(\Omega)$ are separable. Furthermore they are reflexive if $p_{-}>1$.

Denote the dual space of $W_{0}^{k, p(x)}(\Omega)$ by $W^{-k, p^{\prime}(x)}(\Omega)$; then we have the following.

Lemma 6 (see [1]). Let $p \in \mathbf{P}(\Omega) \cap L^{\infty}(\Omega)$. Then for every $G \in W^{-k, p^{\prime}(x)}(\Omega)$, there exists $g_{\alpha} \in\left\{g_{\alpha} \in L^{p^{\prime}(x)}(\Omega):|\alpha| \leq k\right\}$ such that

$$
G(u)=\sum_{|\alpha| \leq k} \int_{\Omega} D^{\alpha} u(x) g_{\alpha}(x) d x, \quad \forall u \in W_{0}^{k, p(x)}(\Omega) .
$$

The norm of $W^{-k, p^{\prime}(x)}(\Omega)$ is defined as

$$
\|G\|_{-k, p^{\prime}(x)}=\sup \left\{\frac{|G(u)|}{\|u\|_{k, p(x)}}: u \in W^{k, p(x)}(\Omega) \backslash\{0\}\right\} .
$$

Lemma 7 (see [4]). Let $\Omega$ be a domain in $\mathbb{R}^{n}$ with cone property. If $p: \bar{\Omega} \rightarrow \mathbb{R}$ is Lipschitz continuous and $1<p_{-} \leq$ $p^{+}<n / k, q: \bar{\Omega} \rightarrow \mathbb{R}$ is measurable and satisfies $p(x) \leq$ $q(x) \leq p^{*}(x):=n p(x) /(n-k p(x))$ for almost every $x \in \bar{\Omega}$; then there is a continuous embedding $W^{k, p(x)}(\Omega) \hookrightarrow L^{q(x)}(\Omega)$.

Lemma 8 (see [4]). Let $\Omega$ be a domain in $\mathbb{R}^{n}$ with cone property. If $p: \bar{\Omega} \rightarrow \mathbb{R}$ is continuous and $1<p_{-} \leq p^{+}<n / k$, then for any measurable function $q(x)$ defined in $\Omega$ with $p(x) \leq$ $q(x)$ for almost every $x \in \bar{\Omega}$ and $\inf _{x \in \bar{\Omega}}\left(p^{*}(x)-q(x)\right)>0$, 
there is a continuous compact embedding $W^{k, p(x)}(\Omega) \hookrightarrow \hookrightarrow$ $L^{q(x)}(\Omega)$.

Lemma 9 (see [6]). Let $p \in \mathbf{P}(\Omega)$; then the Hardy-Littlewood maximal operator is bounded on $L^{p(x)}(\Omega)$.

\section{Young Measure Generated by Sequences in Variable Exponent Space}

Weak convergence is a basic tool of modern nonlinear analysis because it has the same compactness properties as the convergence in finite dimensional spaces (see [21]). But this notion does not behave as we desire with respect to nonlinear functionals and operators. Young measures are a device to overcome these difficulties. For the details we refer to [21-24]. Inspired by these works, we will discuss Young measures in variable exponent spaces. In some references Young measures are called parameterized measures. Here we take the two terms as equivalent. First, we recall the definition and some Lemmas on Young measures.

Definition 10 (see [21]). Assume that the sequence $\left\{f_{k}\right\}_{k=1}^{\infty}$ is bounded in $L^{\infty}\left(\Omega ; \mathbb{R}^{m}\right)$. Then there exist a subsequence $\left\{f_{k_{j}}\right\}_{j=1}^{\infty} \subset\left\{f_{k}\right\}_{k=1}^{\infty}$ and a Borel probability measure $v_{x}$ on $\mathbb{R}^{m}$ for a.e. $x \in \Omega$, such that for each $F \in C\left(\mathbb{R}^{m}\right)$ we have

$$
F\left(f_{k_{j}}\right) \stackrel{*}{\rightarrow} \bar{F} \quad \text { weakly }^{*} \text { in } L^{\infty}(\Omega),
$$

where

$$
\left.\bar{F}(x) \equiv \int_{\mathbb{R}^{m}} F(y) d v_{x}(y) \quad \text { (a.e. } x \in \Omega\right) .
$$

We call $\left\{v_{x}\right\}_{x \in U}$ the family of Young measure associated with the subsequence $\left\{f_{k_{j}}\right\}_{j=1}^{\infty}$.

Lemma 11 (see [22]). Let $\Omega \subset \mathbb{R}^{N}$ be Lebesgue measurable (not necessarily bounded) and $z_{k}: \Omega \rightarrow \mathbb{R}^{m}, k=1,2, \ldots$, be a sequence of Lebesgue measurable functions. Then there exists a subsequence still denoted as $\left\{z_{k}\right\}$ and a family $\left\{v_{x}\right\}_{x \in \Omega}$ of nonnegative Radon measures on $\mathbb{R}^{n}$, such that

(i) $\left\|v_{x}\right\|:=\int d v_{x} \leq 1$ for almost every $x \in \Omega$;

(ii) $\varphi\left(z_{k}\right) \stackrel{*}{\rightarrow} \bar{\varphi}$ weakly ${ }^{*}$ in $L^{\infty}(\Omega)$ for any $\varphi \in C_{0}\left(\mathbb{R}^{m}\right)$, where $\bar{\varphi}(x)=\left\langle v_{x}, \varphi\right\rangle$ and $C_{0}\left(\mathbb{R}^{m}\right)=\left\{\varphi \in C\left(\mathbb{R}^{m}\right)\right.$ : $\left.\lim _{|z| \rightarrow \infty}|\varphi(z)|=0\right\}$;

(iii) if for any $R>0$

$$
\lim _{L \rightarrow \infty} \sup _{k \in \mathbb{N}} \text { meas }\left\{x \in \Omega \cap B(0, R):\left|z_{k}(x)\right| \geq L\right\}=0 .
$$

Then $\left\|v_{x}\right\|=1$ for almost every $x \in \Omega$, and for any measurable $A \subset \Omega$ there holds $\varphi\left(z_{k}\right) \rightarrow \bar{\varphi}=\left\langle v_{x}, \varphi\right\rangle$ weakly in $L^{1}(A)$ for continuous $\varphi$ provided that the sequence $\varphi\left(z_{k}\right)$ is weakly relative compact in $L^{1}(A)$.

Lemma 11 is the fundamental theorem of Young measure. A family $\left\{v_{x}\right\}_{x \in \Omega}$ satisfying (i)-(ii) always exists, and $v_{x}$ is a
Young measure if (14) holds. The proof and complete analysis of Lemma 11 can be found in $[20,23]$. According to the comments there, we give some remarks.

Remark 12. Ball showed in [23] that under hypothesis (14) for any measurable $A \subset \Omega$

$$
\begin{aligned}
& f\left(x, z_{k}(x)\right)-\left\langle v_{x}, f(x, \cdot)\right\rangle \\
& =\int_{\mathbb{R}^{m}} f(x, \lambda) d v_{x}(\lambda) \text { in } L^{1}(A),
\end{aligned}
$$

for every Caratheodory function $f: A \times \mathbb{R}^{m} \rightarrow \mathbb{R}$ such that $\left\{f\left(x, z_{k}(x)\right)\right\}$ is sequentially weakly relative compact in $L^{1}(A)$.

Remark 13. It was shown in [20] that, in order to identify the Young measure associated with a particular sequence of functions $\left\{z_{j}\right\}$, it is enough to check

$$
\varphi\left(z_{j}\right) \stackrel{*}{\rightarrow} \bar{\varphi} \quad \text { in } L^{\infty}(\Omega),
$$

for every $\varphi \in C_{0}\left(\mathbb{R}^{m}\right)$. It is even enough to have

$$
\lim _{j \rightarrow \infty} \int_{\Omega} \xi(x) \varphi\left(z_{j}(x)\right) d x=\int_{\Omega} \xi(x) \int_{\mathbb{R}^{m}} \varphi(\lambda) d v_{x}(\lambda) d x,
$$

for $\xi$ and $\varphi$ belonging to dense, countable subsets of $L^{1}(\Omega)$ and $C_{0}\left(\mathbb{R}^{m}\right)$, respectively.

Lemma 11 has useful applications in nonlinear partial differential equation theory. The following lemmas are useful for us.

Lemma 14 (see [20]). Let $\left\{f_{j}\right\}$ be a uniformly bounded sequence in $L^{1}(\Omega)$. Then there exist a subsequence (not relabeled), a nonincreasing sequence of measurable subsets $\Omega_{n}$ of $\Omega$ with meas $\Omega_{n} \rightarrow 0$ as $n \rightarrow \infty$, and $f \in L^{1}(\Omega)$ such that

$$
f_{j} \rightarrow f \quad \text { in } L^{1}\left(\Omega \backslash \Omega_{n}\right),
$$

for all $n$.

Lemma 15 (see [20]). Let $\left\{z_{j}\right\}$ be a sequence of vector-valued functions with associated Young measure $v=\left\{v_{x}\right\}_{x \in \Omega}$. If, for $\varphi_{0}$, a nonnegative Carathéodory function, we have

$$
\lim _{j \rightarrow \infty} \int_{\Omega} \varphi_{0}\left(x, z_{j}(x)\right) d x=\int_{\Omega} \int_{\mathbb{R}^{m}} \varphi_{0}(x, \lambda) d v_{x}(\lambda) d x<\infty,
$$

then

$$
\lim _{j \rightarrow \infty} \int_{E} \varphi\left(x, z_{j}(x)\right) d x=\int_{E} \int_{\mathbb{R}^{m}} \varphi_{0}(x, \lambda) d \nu_{x}(\lambda) d x<\infty,
$$

for any measurable subset $E \subset \Omega$ and for any $\varphi$ in the space

$$
\begin{gathered}
\mathscr{E}^{\varphi_{0}}=\{\varphi: \varphi \text { is a Carathéodory function with } \\
\left.|\varphi| \leq C\left(1+\varphi_{0}\right)\right\} .
\end{gathered}
$$


Lemma 16 (see [20]). If $\left\{z_{i}\right\}$ is a sequence of measurable functions with associated parameterized measure $\nu=\left\{v_{x}\right\}_{x \in \Omega}$,

$$
\liminf _{j \rightarrow \infty} \int_{E} \psi\left(x, z_{j}(x)\right) d x \geq \int_{E} \int_{\mathbb{R}^{m}} \psi(x, \lambda) d v_{x}(\lambda) d x,
$$

for every nonnegtive Carathéodory function $\psi$ and every measurable subset $E \subset \Omega$.

Lemma 17 (see [25]). If the sequence $\left\{u_{j}\right\}$ is bounded in $L^{p(x)}\left(\Omega, \mathbb{R}^{m}\right)$, then there is a Young measure $v_{x}$ generated by $\left\{u_{j}\right\}$ satisfying $\left\|v_{x}\right\|=1$, and the weak $L^{1}$-limit of $\left\{u_{j}\right\}$ is $\int_{\mathbb{R}^{m}} \lambda d \nu_{x}(\lambda)$.

Lemma 18 (see [25]). Let meas $\Omega<\infty$. If $u_{k} \rightarrow u$ in $W_{0}^{1, p(x)}\left(\Omega, \mathbb{R}^{m}\right)$, then the sequence $\left\{\left(u_{k}, D u_{k}\right)\right\}$ generates the Young measure $\delta_{u(x)} \otimes v_{x}$. Moreover, for almost every $x \in \Omega$, $v_{x}$ is a probability measure and satisfies $\left\langle v_{x}, I\right\rangle=D u(x)$.

By Lemmas 3.8 and 3.9 in [25], we know that if a sequence $\left\{u_{j}\right\}$ is bounded in $W^{1, p(x)}\left(\Omega, \mathbb{R}^{m}\right)$, then $\left\{\nabla u_{j}\right\}$ can generate a family of Young measures. Now we can define $W^{1, p(x)}$-Young measure for a sequence of functions in $W^{1, p(x)}\left(\Omega, \mathbb{R}^{m}\right)$.

Definition 19. $\left\{v_{x}\right\}_{x \in \Omega}$ is a family of probability measures supported in $\mathbb{M}^{m \times N} .\left\{v_{x}\right\}_{x \in \Omega}$ is called a $W^{1, p(x)}$-Young measure if it can be generated by gradients of a bounded sequence of functions in $W^{1, p(x)}\left(\Omega, \mathbb{R}^{m}\right)$.

Theorem 20. Assume that the sequences $\left\{z_{j}\right\}$ and $\left\{w_{j}\right\}$ are bounded in $L^{p(x)}\left(\Omega, \mathbb{R}^{m}\right)$. We have

(a) if meas $\left\{z_{j} \neq w_{j}\right\} \rightarrow 0$ as $j \rightarrow \infty$, then the Young measures for both sequences are the same;

(b) if $\left\|z_{j}-w_{j}\right\|_{L^{p(x)}\left(\Omega, \mathbb{R}^{m}\right)} \rightarrow 0$ as $j \rightarrow \infty$, then the Young measures for both sequences are the same.

Proof. (a) By Remark 13, it suffices to prove that the limits of $\int_{\Omega} \xi(x) \varphi\left(z_{j}(x)\right) d x$ and $\int_{\Omega} \xi(x) \varphi\left(w_{j}(x)\right) d x$ are the same for every $\xi \in L^{1}(\Omega)$ and every $\varphi \in C_{0}\left(\mathbb{R}^{m}\right)$. We conclude the result from

$$
\begin{aligned}
& \left|\int_{\Omega} \xi(x) \varphi\left(z_{j}(x)\right) d x-\int_{\Omega} \xi(x) \varphi\left(w_{j}(x)\right) d x\right| \\
& =\mid \int_{\left\{z_{j} \neq w_{j}\right\}} \xi(x) \varphi\left(z_{j}(x)\right) d x \\
& \quad-\int_{\left\{z_{j} \neq w_{j}\right\}} \xi(x) \varphi\left(w_{j}(x)\right) d x \mid \\
& \leq\left|\int_{\left\{z_{j} \neq w_{j}\right\}} \xi(x) \varphi\left(z_{j}(x)\right) d x\right| \\
& \quad+\left|\int_{\left\{z_{j} \neq w_{j}\right\}} \xi(x) \varphi\left(w_{j}(x)\right) d x\right| \\
& \leq \int_{\left\{z_{j} \neq w_{j}\right\}} 2\|\varphi\|_{L^{\infty}(\Omega)}|\xi(x)| d x \longrightarrow 0,
\end{aligned}
$$

as $j \rightarrow \infty$. (b) For any $\xi \in L^{1}(\Omega)$ and $\varphi \in C_{0}\left(\mathbb{R}^{m}\right)$, we have

$$
\left|\xi(x)\left(\varphi\left(z_{j}(x)\right)-\varphi\left(w_{j}(x)\right)\right)\right| \leq 2\|\varphi\|_{L^{\infty}(\Omega)}|\xi(x)| .
$$

Since $\left\|z_{j}-w_{j}\right\|_{L^{p(x)}\left(\Omega, \mathbb{R}^{m}\right)} \rightarrow 0$, as $j \rightarrow \infty$, there exists a subsequence still denoted as $\left\{z_{j}-w_{j}\right\}$ such that $z_{j}-w_{j} \rightarrow 0$, for almost every $x \in \Omega$, as $j \rightarrow \infty$. Then $\varphi\left(z_{j}(x)\right)-$ $\varphi\left(w_{j}(x)\right) \rightarrow 0$, for almost every $x \in \Omega$, as $j \rightarrow \infty$. By dominated convergence theorem, we get

$$
\lim _{j \rightarrow \infty} \int_{\Omega} \xi(x) \varphi\left(z_{j}(x)\right) d x=\lim _{j \rightarrow \infty} \int_{\Omega} \xi(x) \varphi\left(w_{j}(x)\right) d x .
$$

Example 21. Assume that $\left\{z_{j}\right\}$ is a bounded sequence in $L^{p(x)}\left(\Omega, \mathbb{R}^{m}\right)$ and let $v=\left\{v_{x}\right\}_{x \in \Omega}$ be its associated Young measure. Consider the truncation operators

$$
T_{k}(s)= \begin{cases}s, & |s|<k, \\ \frac{k s}{|s|}, & |s| \geq k .\end{cases}
$$

By Lemma 2 , there exists a constant $C$ such that $\rho_{p(x)}\left(z_{j}\right) \leq C$. Then for any subsequence $k(j) \rightarrow \infty$ as $j \rightarrow \infty$,

$$
\left|\left\{\left|z_{j}\right| \geq k(j)\right\}\right| \leq \frac{C}{k(j)^{p_{-}}} \longrightarrow 0 .
$$

Thus the Young measure corresponding to $T_{k(j)}\left(z_{j}\right)$ is also $\nu$.

Theorem 22. Let $\left\{v_{j}\right\}$ be a bounded sequence in $W^{1, p(x)}\left(\Omega, \mathbb{R}^{m}\right)$ such that the sequence $\left\{\nabla v_{j}\right\}$ generates the Young measure $v=\left\{v_{x}\right\}_{x \in \Omega}$. Then we have

(a) there exists $u \in W^{1, p(x)}\left(\Omega, \mathbb{R}^{m}\right)$ such that $\nabla u(x)=$ $\int_{\mathbb{M}^{m \times N}} \lambda d v_{x}(\lambda) \in \mathbb{M}^{m \times N}$

(b) there exists a new sequence $\left\{u_{k}\right\}$ bounded in $W^{1, p(x)}\left(\Omega, \mathbb{R}^{m}\right)$ such that $\left\{\nabla u_{k}\right\}$ generates the same Young measure $v$ and $u_{k}-u \in W_{0}^{1, p(x)}\left(\Omega, \mathbb{R}^{m}\right)$ for all $k$. Moreover if $\left\{\left|\nabla v_{j}\right|^{p(x)}\right\}$ is equi-integrable, so is $\left\{\left|\nabla u_{k}\right|^{p(x)}\right\}$.

Proof. (a) Since $\left\{v_{j}\right\}$ is a bounded sequence in $W^{1, p(x)}\left(\Omega, \mathbb{R}^{m}\right)$, by Lemma 5 , there exists a subsequence still denoted as $\left\{v_{j}\right\}$ such that $v_{j} \rightarrow u$ in $W^{1, p(x)}\left(\Omega, \mathbb{R}^{m}\right)$ for some $u \in$ $W^{1, p(x)}\left(\Omega, \mathbb{R}^{m}\right)$. Moreover $\nabla v_{j} \rightarrow \nabla u$ in $L^{p(x)}\left(\Omega, \mathbb{M}^{m \times N}\right) \subset$ $L^{1}\left(\Omega, \mathbb{M}^{m \times N}\right)$. By Lemmas 17 and $18, \nabla v_{j} \rightarrow \int_{\mathbb{M}^{m \times N}} \lambda d v_{x}(\lambda)$ in $L^{1}\left(\Omega, \mathbb{M}^{m \times N}\right)$. Thus $\nabla u(x)=\int_{\mathbb{M}^{m \times N}} \lambda d v_{x}(\lambda)$.

(b) Let $\left\{\eta_{k}\right\}$ be a sequence of cut-off functions satisfying

(i) $\eta_{k}=1$ on $\partial \Omega$;

(ii) $\eta_{k}=0$ in $\Omega_{k}=\{x \in \Omega: \operatorname{dist}(x, \partial \Omega) \geq 1 / k\}$;

(iii) $\left|\nabla \eta_{k}\right| \leq C k$ for some constant $C$. 
Consider the sequence $\left\{w_{j k}\right\}$

$$
w_{j k}(x)=\eta_{k}(x) u(x)+\left(1-\eta_{k}(x)\right) v_{j}(x) .
$$

Clearly $w_{j k}-u \in W_{0}^{1, p(x)}\left(\Omega, \mathbb{R}^{m}\right)$ for all $j, k$, and

$$
\begin{aligned}
\nabla w_{j k}(x)= & \eta_{k}(x) \nabla u(x)+\left(1-\eta_{k}(x)\right) \nabla v_{j}(x) \\
& +\left(u(x)-v_{j}(x)\right) \cdot \nabla \eta_{k}(x) .
\end{aligned}
$$

Notice that

$$
\begin{gathered}
\int_{\Omega}\left|\eta_{k}(x) \nabla u(x)\right|^{p(x)} d x \leq \int_{\Omega}|\nabla u(x)|^{p(x)} d x, \\
\int_{\Omega}\left|\left(1-\eta_{k}(x)\right) \nabla v_{j}(x)\right|^{p(x)} d x \leq \int_{\Omega}\left|\nabla v_{j}(x)\right|^{p(x)} d x, \\
\int_{\Omega}\left|u(x)-v_{j}(x)\right|^{p(x)}\left|\nabla \eta_{k}(x)\right|^{p(x)} d x \\
\leq \max \left\{(C k)^{p_{-}},(C k)^{p_{+}}\right\} \int_{\Omega}\left|u(x)-v_{j}(x)\right|^{p(x)} d x .
\end{gathered}
$$

Lemma 8 implies that $\left\|v_{j}-u\right\|_{L^{p(x)}\left(\Omega, \mathbb{R}^{m}\right)} \rightarrow 0$ as $j \rightarrow 0$. Then we can choose a subsequence $\{j(k)\}$ so that $\left\{\left|\nabla w_{j(k), k}\right|^{p(x)}\right\}$ is bounded in $L^{1}(\Omega)$ and equi-integrable if $\left\{\left|\nabla v_{j}\right|^{p(x)}\right\}$ is equiintegrable. Let $\left\{u_{k}\right\}$ be the sequence $\left\{w_{j(k), k}\right\}$. Moreover

$$
\left|\left\{\nabla v_{j}(x) \neq \nabla w_{j(k), k}\right\}\right|=\left|\left\{\eta_{k}>0\right\}\right| \longrightarrow 0, \quad \text { as } k \longrightarrow \infty \text {. }
$$

Thus by Theorem 20, $\left\{\nabla u_{k}\right\}$ and $\left\{\nabla v_{j}\right\}$ generate the same Young measure.

Theorem 23. Let $\left\{v_{j}\right\}$ be a bounded sequence in $W^{1, p(x)}\left(\Omega, \mathbb{R}^{m}\right)$. Then there always exists another sequence $\left\{u_{j}\right\}$ of Lipschitz functions with $u_{j} \in W^{1, \infty}\left(\Omega, \mathbb{R}^{m}\right)$ for all $j$ such that $\left\{\left|\nabla u_{j}\right|^{p(x)}\right\}$ is equi-integrable and the two sequences of gradients, $\left\{\nabla v_{j}\right\}$ and $\left\{\nabla u_{j}\right\}$, generate the same $W^{1, p(x)}$-Young measure.

Proof. By Lemma 6, we can assume that $v_{j} \rightarrow u$ in $W^{1, p(x)}\left(\Omega, \mathbb{R}^{m}\right)$ for some $u \in W^{1, p(x)}\left(\Omega, \mathbb{R}^{m}\right)$. By Theorem 22 , we can assume that $v_{j}-u \in W_{0}^{1, p(x)}\left(\Omega, \mathbb{R}^{m}\right)$. Extend $w_{j}=v_{j}-$ $u$ by 0 to all of $\mathbb{R}^{N}$. By density, we can find $z_{j} \in C_{0}^{\infty}\left(\mathbb{R}^{N}, \mathbb{R}^{m}\right)$ such that $\left\|z_{j}-w_{j}\right\|_{W^{1, p(x)}\left(\mathbb{R}^{N}, \mathbb{R}^{m}\right)} \rightarrow 0$ as $j \rightarrow \infty$. This implies that $\left\{z_{j}\right\}$ is a bounded sequence in $W^{1, p(x)}\left(\Omega, \mathbb{R}^{m}\right)$. Consider the sequence $\left\{M^{*}\left(z_{j}\right)\right\}$, where

$$
M^{*}\left(z_{j}\right)=M\left(\left|z_{j}\right|\right)+M\left(\left|\nabla z_{j}\right|\right)
$$

and $M$ is the Hardy-Littlewood maximal operator. By Lemma 9, $\left\{M^{*}\left(z_{j}\right)\right\}$ is bounded in $L^{p(x)}\left(\mathbb{R}^{N}\right)$. Let $\mu=$ $\left\{\mu_{x}\right\}_{x \in \mathbb{R}^{N}}$ be the corresponding Young measure (possibly for an appropriate subsequence). For fixed $k,\left\{T_{k} M^{*}\left(z_{j}\right)\right\}$ is bounded in $L^{\infty}\left(\mathbb{R}^{N}\right)$ and for any $E \subset \mathbb{R}^{N}$,

$$
\int_{E}\left|T_{k} M^{*}\left(z_{j}\right)\right|^{p(x)} d x \leq \int_{E} k^{p(x)} d x \leq k^{p_{+}} \text {meas } E .
$$

We can get by Dunford-Pettis and Lemma 11

$$
\lim _{j \rightarrow \infty} \int_{\mathbb{R}^{N}}\left|T_{k} M^{*}\left(z_{j}\right)\right|^{p(x)} d x=\int_{\mathbb{R}^{N}} \int_{\mathbb{R}}\left|T_{k}(x)\right|^{p(x)} d \mu_{x}(s) d x .
$$

By Levei theorem, we have

$$
\begin{aligned}
\lim _{k \rightarrow \infty} \int_{\mathbb{R}}\left|T_{k}(s)\right|^{p(x)} d \mu_{x}(s) & =\int_{\mathbb{R}} \lim _{k \rightarrow \infty}\left|T_{k}(s)\right|^{p(x)} d \mu_{x}(s) \\
& =\int_{\mathbb{R}}|s|^{p(x)} d \mu_{x}(s):=g(x)
\end{aligned}
$$

and $g(x) \in L^{1}\left(\mathbb{R}^{N}\right)$. Moreover

$$
\lim _{k \rightarrow \infty} \int_{\mathbb{R}^{N}} \int_{\mathbb{R}}\left|T_{k}(s)\right|^{p(x)} d \mu_{x}(s) d x=\int_{\mathbb{R}^{N}} g(x) d x .
$$

Thus we can get

$$
\begin{aligned}
\lim _{k \rightarrow \infty} & \lim _{j \rightarrow \infty} \int_{\mathbb{R}^{N}}\left|T_{k} M^{*}\left(z_{j}\right)\right|^{p(x)} d x \\
\quad= & \lim _{k \rightarrow \infty} \int_{\mathbb{R}^{N}} \int_{\mathbb{R}}\left|T_{k}(s)\right|^{p(x)} d \mu_{x}(s) d x \\
& =\int_{\mathbb{R}^{N}} g(x) d x .
\end{aligned}
$$

And we can find a subsequence $k(j) \rightarrow \infty$ as $j \rightarrow \infty$ such that

$$
\lim _{j \rightarrow \infty} \int_{\mathbb{R}^{N}}\left|T_{k(j)} M^{*}\left(z_{j}\right)\right|^{p(x)} d x=\int_{\mathbb{R}^{N}} g(x) d x .
$$

By Lemma 15 , let $\varphi_{0}(x, \lambda)=|\lambda|^{p(x)}$. For any $\xi \in L^{\infty}\left(\mathbb{R}^{N}\right)$, there exists a constant $C$ such that $|\xi(x)| \leq C$. We can take $\varphi(x, \lambda)=\xi(x)|\lambda|^{p(x)} \leq C\left(1+\varphi_{0}\right)$. Thus

$$
\begin{aligned}
\int_{\mathbb{R}^{N}} \xi(x)\left|T_{k(j)} M^{*}\left(z_{j}\right)\right|^{p(x)} d x \\
\quad \longrightarrow \int_{\mathbb{R}^{N}} \int_{\mathbb{R}} \xi(x)|s|^{p(x)} d \mu_{x}(s) d x \\
=\int_{\mathbb{R}^{N}} \xi(x) g(x) d x .
\end{aligned}
$$

That is, $\left|T_{k(j)} M^{*}\left(z_{j}\right)\right|^{p(x)} \rightarrow g$ in $L^{1}\left(\mathbb{R}^{N}\right)$. Let $A_{j}=\left\{M^{*}\left(z_{j}\right) \geq\right.$ $k(j)\}$. Since $\left\{M^{*}\left(z_{j}\right)\right\}$ is bounded in $L^{p(x)}\left(\mathbb{R}^{N}\right)$ and $k(j) \rightarrow$ $\infty$ as $j \rightarrow \infty,\left|A_{j}\right| \rightarrow 0$. There exists Lipschitz function $\bar{u}_{j}$, such that $\bar{u}_{j}=z_{j}$ in $\mathbb{R}^{N} \backslash A_{j}$ and $\left|\nabla \bar{u}_{j}\right| \leq C(N) k(j)$ for all $j$. Because $\left\{\nabla \bar{u}_{j} \neq \nabla z_{j}\right\} \subset A_{j}$, by Theorem 20 again, $\left\{\nabla \bar{u}_{j}\right\}$ and $\left\{\nabla z_{j}\right\}$ generate the same Young measure. Since $M^{*}\left(z_{j}\right) \geq$ $\left|\nabla z_{j}\right|$, we have

$$
\left|\nabla \bar{u}_{j}\right|^{p(x)} \leq \max \left\{C(N)^{p_{-}}, C(N)^{p_{+}}\right\}\left|T_{k(j)} M^{*}\left(z_{j}\right)\right|^{p(x)} .
$$

It follows from the equi-integrability of $\left\{\left|T_{k(j)} M^{*}\left(z_{j}\right)\right|^{p(x)}\right\}$ that $\left\{\left|\nabla \bar{u}_{j}\right|^{p(x)}\right\}$ is equi-integrable. Take $u_{j}=\left.\bar{u}_{j}\right|_{\Omega}+u$. Then the sequence $\left\{u_{j}\right\}$ verifies the conclusion of the theorem. 
Theorem 24. Let $\Pi=\left\{\Pi_{(x, y)}\right\}$ be a family of probability measures supported in $\mathbb{M}^{m \times N} \times \mathbb{M}^{m \times N}$. $\Pi$ is the Young measure generated by a sequence $\left\{g_{j}(x, y)\right\}=\left\{\left(\nabla u_{j}(x), \nabla u_{j}(y)\right)\right\}$, where $\left\{u_{j}\right\}$ is a bounded sequence in $W^{1, p(x)}\left(\Omega, \mathbb{R}^{m}\right)$. Then

$$
\Pi_{(x, y)}=v_{x} \otimes v_{y}, \quad(x, y) \in \Omega \times \Omega,
$$

where $v=\left\{v_{x}\right\}_{x \in \Omega}$ is the Young measure generated by the sequence of gradients $\left\{\nabla u_{j}\right\}$.

Proof. Let $\varphi=\varphi_{1} \varphi_{2}$ and $\theta=\theta_{1} \theta_{2}$. We have

$$
\begin{aligned}
& \int_{\Omega \times \Omega} \theta_{1}(x) \theta_{2}(y) \\
& \quad \times\left(\int_{\mathbb{M}^{m \times N} \times \mathbb{M}^{m \times N}} \varphi_{1}\left(\lambda_{1}\right) \varphi_{2}\left(\lambda_{2}\right) d \Pi_{(x, y)}\left(\lambda_{1}, \lambda_{2}\right)\right) d x d y \\
& =\lim _{j \rightarrow \infty} \int_{\Omega \times \Omega} \theta_{1}(x) \theta_{2}(y) \varphi\left(g_{j}(x, y)\right) d x d y \\
& =\lim _{j \rightarrow \infty} \int_{\Omega \times \Omega} \theta_{1}(x) \theta_{2}(y) \varphi_{1}\left(\nabla u_{j}(x)\right) \\
& =\lim _{j \rightarrow \infty}\left(\int_{\Omega} \theta_{1}(x) \varphi_{1}\left(\nabla u_{j}(x)\right) d x\right) \\
& \quad \times\left(\int_{\Omega} \theta_{2}(y) \varphi_{2}\left(\nabla u_{j}(y)\right) d y\right) \\
& =\int_{\Omega} \theta_{1}(x)\left(\int_{\mathbb{M}^{m \times N}} \varphi_{1}\left(\lambda_{1}\right) d v_{x}\left(\lambda_{1}\right)\right) d x \\
& \quad \times \int_{\Omega} \theta_{2}(x)\left(\int_{\mathbb{M}^{m \times N}} \varphi_{2}\left(\lambda_{2}\right) d v_{y}\left(\lambda_{2}\right)\right) d y \\
& =\int_{\Omega \times \Omega} \theta_{1}(x) \theta_{2}(y) \\
& \quad \times \int_{\mathbb{M}^{m \times N} \times \mathbb{M}^{m \times N}} \varphi_{1}\left(\lambda_{1}\right) \varphi_{2}\left(\lambda_{2}\right) d\left(v_{x} \otimes v_{y}\right)\left(\lambda_{1}, \lambda_{2}\right) d x d y .
\end{aligned}
$$

Remark 25. Concerning the above result it must be pointed out that we have the representation

$$
\begin{aligned}
& \lim _{j \rightarrow \infty} \int_{\Omega \times \Omega} \psi\left(\nabla u_{j}(x), \nabla u_{j}(y)\right) d x d y \\
& \quad=\int_{\Omega \times \Omega} \int_{\mathbb{M}^{m \times N} \times \mathbb{M}^{m \times N}} \psi\left(\lambda_{1}, \lambda_{2}\right) d v_{x}\left(\lambda_{1}\right) d v_{y}\left(\lambda_{2}\right) d x d y
\end{aligned}
$$

for any continuous $\psi$ such that $\left\{\psi\left(\nabla u_{j}(x), \nabla u_{j}(y)\right)\right\}_{j}$ converges weakly in $L^{1}(\Omega \times \Omega)$.

\section{Weak Lower Semicontinuity and Relaxation}

Theorem 26. Assume that (2) holds. $J$ is weakly lower semicontinuous in $W^{1, p(x)}\left(\Omega, \mathbb{R}^{m}\right)$ if and only if for any $W^{1, p(x)}$. Young measure $v=\left\{v_{x}\right\}_{x \in \Omega}$,

$$
\begin{aligned}
& \iint_{\Omega} W\left(x, y, \int_{\mathbb{M}^{m \times N}} \lambda_{1} d v_{x}\left(\lambda_{1}\right), \int_{\mathbb{M}^{m \times N}} \lambda_{2} d v_{y}\left(\lambda_{2}\right)\right) d x d y \\
& \quad \leq \iint_{\Omega} \iint_{\mathbb{M}^{m \times N}} W\left(x, y, \lambda_{1}, \lambda_{2}\right) d v_{x}\left(\lambda_{1}\right) d v_{y}\left(\lambda_{2}\right) d x d y .
\end{aligned}
$$

Proof. For any $W^{1, p(x)}$-Young measure $v$, by Definition 19, $v$ can be generated by a bounded sequence $\left\{u_{j}\right\}$ in $W^{1, p(x)}\left(\Omega, \mathbb{R}^{m}\right)$. Moreover we can assume that $u_{j} \rightarrow u$ in $W^{1, p(x)}\left(\Omega, \mathbb{R}^{m}\right)$ (possibly for a suitable subsequence). By Lemma 18, we have $\nabla u(x)=\int_{\mathbb{M}^{m \times N}} \lambda d v_{x}(\lambda)$. According to Theorem 22, there exists another sequence $\left\{\bar{u}_{j}\right\}$ such that $\left\{\left|\nabla \bar{u}_{j}\right|^{p(x)}\right\}$ is weakly convergent in $L^{1}(\Omega) .\left\{\nabla \bar{u}_{j}\right\}$ and $\left\{\nabla u_{j}\right\}$ generate the same Young measure. Going if necessary to a subsequence, we can assume that $\left\{\bar{u}_{j}\right\}$ is also weakly convergent to $u$. Actually if $\bar{u}_{j} \rightarrow \bar{u} \neq u$, by Lemma 18 , we have $\nabla \bar{u}=\int_{\mathbb{M}^{m \times N}} \lambda d v_{x}(\lambda)=\nabla u$. Then we can take $\bar{u}_{j}-\bar{u}+u \rightarrow u$, and $\left\{\left|\nabla\left(w_{j}-\bar{u}+u\right)\right|^{p(x)}\right\}$ is also weakly convergent in $L^{1}(\Omega)$. Since $J(u)$ is weakly lower semicontinuous and (2) holds, we can get

$$
\begin{aligned}
& \iint_{\Omega} W\left(x, y, \int_{\mathbb{M}^{m \times N}} \lambda_{1} d v_{x}\left(\lambda_{1}\right)\right. \\
& \left.\quad \int_{\mathbb{M}^{m \times N}} \lambda_{2} d v_{y}\left(\lambda_{2}\right)\right) d x d y \\
& \quad=\iint_{\Omega} W(x, y, \nabla u(x), \nabla u(y)) d x d y \\
& \quad \leq \lim _{j \rightarrow \infty} \iint_{\Omega} W\left(x, y, \bar{u}_{j}(x), \bar{u}_{j}(y)\right) d x d y \\
& \quad=\iint_{\Omega} \iint_{\mathbb{M}^{m \times N}} W\left(x, y, \lambda_{1}, \lambda_{2}\right) d v_{x}\left(\lambda_{1}\right) d v_{y}\left(\lambda_{2}\right) d x d y .
\end{aligned}
$$

On the other hand, if $u_{j} \rightarrow u$ in $W^{1, p(x)}\left(\Omega, \mathbb{R}^{m}\right)$, then by Lemma $18\left\{u_{j}\right\}$ can generate a family of $W^{1, p(x)}$-Young measure $v=\left\{v_{x}\right\}_{x \in \Omega}$ and $\nabla u(x)=\int_{\mathbb{M}^{m \times N}} \lambda d v_{x}(\lambda)$. Thus (2) and Lemma 16 imply that

$$
\begin{aligned}
& \lim _{j \rightarrow \infty} \iint_{\Omega} W\left(x, y, \nabla u_{j}(x), \nabla u_{j}(y)\right) d x d y \\
& \quad \geq \iint_{\Omega} \iint_{\mathbb{M}^{m \times N}} W\left(x, y, \lambda_{1}, \lambda_{2}\right) d v_{x}\left(\lambda_{1}\right) d v_{y}\left(\lambda_{2}\right) d x d y
\end{aligned}
$$




$$
\begin{array}{r}
\geq \iint_{\Omega} W\left(x, y, \int_{\mathbb{M}^{m \times N}} \lambda_{1} d v_{x}\left(\lambda_{1}\right),\right. \\
\left.\int_{\mathbb{M}^{m \times N}} \lambda_{2} d v_{y}\left(\lambda_{2}\right)\right) d x d y \\
=\iint_{\Omega} W(x, y, \nabla u(x), \nabla u(y)) d x d y .
\end{array}
$$

Theorem 27. Let $u_{j} \rightarrow u$ in $W^{1, p(x)}\left(\Omega, \mathbb{R}^{m}\right)$ and $v=\left\{v_{x}\right\}_{x \in \Omega}$ be the $W^{1, p(x)}$-Young measure associated to $\left\{\nabla u_{j}\right\}$ (or possibly to a subsequence). Then

$$
\begin{aligned}
& \iint_{E} W(x, y, \nabla u(x), \nabla u(y)) d x d y \\
& \leq \liminf _{j \rightarrow \infty} \iint_{E} W\left(x, y, \nabla u_{j}(x), \nabla u_{j}(y)\right) d x d y,
\end{aligned}
$$

for all measurable $E \subset \Omega$, if and only if

$$
\begin{array}{r}
W(x, y, \nabla u(x), \nabla u(y)) \\
\leq \iint_{\mathbb{M}^{m \times N}} W\left(x, y, \lambda_{1}, \lambda_{2}\right) d v_{x}\left(\lambda_{1}\right) d v_{y}\left(\lambda_{2}\right), \\
\text { a.e. }(x, y) \in \Omega \times \Omega .
\end{array}
$$

Proof. In view of Theorem 26, we only need show the necessity. By Lemma 14, there exists a sequence of measurable sets $\left\{\Omega_{k}\right\}$ such that $\Omega_{k+1} \subset \Omega_{k}$ for each $k, \lim _{k \rightarrow \infty}\left|\Omega_{k}\right|=0$, and

$$
\begin{aligned}
& W\left(x, y, \nabla u_{j}(x), \nabla u_{j}(y)\right) \\
& \quad-\iint_{\mathbb{M}^{m \times N}} W\left(x, y, \lambda_{1}, \lambda_{2}\right) d v_{x}\left(\lambda_{1}\right) d v_{y}\left(\lambda_{2}\right)
\end{aligned}
$$

in $L^{1}\left(\left(\Omega \backslash \Omega_{k}\right) \times\left(\Omega \backslash \Omega_{k}\right)\right)$ for all $k$. By (47) and Lemma 11

$$
\begin{aligned}
& \iint_{E \backslash \Omega_{k}} W(x, y, \nabla u(x), \nabla u(y)) d x d y \\
& \quad \leq \lim _{j \rightarrow \infty} \iint_{E \backslash \Omega_{k}} W\left(x, y, \nabla u_{j}(x), \nabla u_{j}(y)\right) d x d y \\
& \quad=\iint_{E \backslash \Omega_{k}} \iint_{\mathbb{M}^{m \times N}} W\left(x, y, \lambda_{1}, \lambda_{2}\right) d v_{x}\left(\lambda_{1}\right) d v_{y}\left(\lambda_{2}\right) d x d y .
\end{aligned}
$$

Lemma 16 implies that $\int_{\mathbb{M}^{m \times N}} \int_{\mathbb{M}^{m \times N}} W\left(x, y, \lambda_{1}\right.$, $\left.\lambda_{2}\right) d \nu_{x}\left(\lambda_{1}\right) d \nu_{y}\left(\lambda_{2}\right) \in L^{1}(\Omega \times \Omega)$ and by (47) $W(x, y$, $\nabla u(x), \nabla u(y)) \in L^{1}(\Omega \times \Omega)$. Therefore

$$
\begin{gathered}
\lim _{k \rightarrow \infty} \iint_{E \backslash \Omega_{k}} \iint_{\mathbb{M}^{m \times N}} W\left(x, y, \lambda_{1}, \lambda_{2}\right) d v_{x}\left(\lambda_{1}\right) d v_{y}\left(\lambda_{2}\right) d x d y \\
\quad=0 \\
\quad \lim _{k \rightarrow \infty} \iint_{E \backslash \Omega_{k}} W(x, y, \nabla u(x), \nabla u(y)) d x d y=0 .
\end{gathered}
$$

Moreover

$$
\begin{aligned}
& \iint_{E} W(\nabla u(x), \nabla u(y)) d x d y \\
& \quad \leq \iint_{E} \iint_{\mathbb{M}^{m \times N}} W\left(x, y, \lambda_{1}, \lambda_{2}\right) d \nu_{x}\left(\lambda_{1}\right) d \nu_{y}\left(\lambda_{2}\right) d x d y .
\end{aligned}
$$

Then we can get the conclusion.

In many models from mathematical physics we need to consider variational integrals like

$$
\begin{array}{r}
J(u)=\iint_{\Omega} W(x, y, \nabla u(x), \nabla u(y)) d x d y, \\
u \in W^{1, p(x)}\left(\Omega, \mathbb{R}^{m}\right), \quad u-u_{0} \in W_{0}^{1, p(x)}\left(\Omega, \mathbb{R}^{m}\right),
\end{array}
$$

where $u_{0}$ is some fixed function in $W^{1, p(x)}\left(\Omega, \mathbb{R}^{m}\right)$. The energy of a Young measure is defined by

$$
\bar{J}(\mu)=\iint_{\mathbb{M}^{m \times N}} W\left(x, y, \lambda_{1}, \lambda_{2}\right) d \mu_{x}\left(\lambda_{1}\right) d \mu_{y}\left(\lambda_{2}\right),
$$

where $\mu$ is a $W^{1, p(x)}$-Young measure generated by a sequence of gradients in $W^{1, p(x)}\left(\Omega, \mathbb{R}^{m}\right)$; there exists $u \in$ $W^{1, p(x)}\left(\Omega, \mathbb{R}^{m}\right)$, such that $\nabla u(x)=\int_{\mathbb{M}^{m \times N}} \lambda d \mu_{x}(\lambda), u-u_{0} \in$ $W_{0}^{1, p(x)}\left(\Omega, \mathbb{R}^{m}\right)$. Let

$$
\begin{gathered}
\mathscr{A}=\left\{u \in W^{1, p(x)}\left(\Omega, \mathbb{R}^{m}\right): u-u_{0} \in W_{0}^{1, p(x)}\left(\Omega, \mathbb{R}^{m}\right)\right\}, \\
\overline{\mathscr{A}}=\left\{u \text { is a } W^{1, p(x)}\right. \text {-Young measure : } \\
\exists u \in W^{1, p(x)}\left(\Omega, \mathbb{R}^{m}\right), \\
\nabla u(x)=\int_{\mathbb{M}^{m \times N}} \lambda d \mu_{x}(\lambda), \\
\left.u-u_{0} \in W_{0}^{1, p(x)}\left(\Omega, \mathbb{R}^{m}\right)\right\} .
\end{gathered}
$$

Theorem 28. Let

$$
\begin{aligned}
& m=\inf \{J(u): u \in \mathscr{A}\}, \\
& \bar{m}=\inf \{\bar{J}(\mu), \mu \in \overline{\mathscr{A}}\} .
\end{aligned}
$$

Then $m=\bar{m}$ and $\bar{m}$ is indeed a minimum.

Proof. Consider the following steps.

Step 1. Equation (56) admits a minimizing sequence $\left\{u_{j}\right\}$ such that $\left\{\left|\nabla u_{j}\right|^{p(x)}\right\}$ is weakly convergent in $L^{1}(\Omega)$.

Let $\left\{v_{j}\right\}$ be a minimizing sequence for (56). By (2), $\left\{v_{j}\right\}$ is a bounded sequence in $W^{1, p(x)}\left(\Omega, \mathbb{R}^{m}\right)$. By Lemma 17 , let $\nu=$ $\left\{v_{x}\right\}_{x \in \Omega}$ denote the Young measure associated to the sequence of gradients. According to Theorem 22, there exists another 
sequence $\left\{w_{j}\right\}$ such that $\left\{\left|\nabla w_{j}\right|^{p(x)}\right\}$ is weakly convergent in $L^{1}(\Omega)$ and $\left\{\nabla w_{j}\right\}$ and $\left\{\nabla v_{j}\right\}$ generate the same Young measure. Going if necessary to a subsequence, we can assume that both $v_{j}$ and $w_{j}$ are weakly convergent to $u$. If $w_{j} \rightarrow w \neq u$, by Lemma 18, we have $\nabla w=\int_{\mathbb{M}^{m \times N}} A d v_{x}(A)=\nabla u$. Then we can take $w_{j}-w+u \rightarrow u$, and $\left\{\left|\nabla\left(w_{j}-w+u\right)\right|^{p(x)}\right\}$ is also weakly convergent in $L^{1}(\Omega)$. By Theorem 22, there exists a sequence $\left\{u_{j}\right\}$ in $W^{1, p(x)}\left(\Omega, \mathbb{R}^{m}\right)$ such that $u_{j}-u \in W_{0}^{1, p(x)}\left(\Omega, \mathbb{R}^{m}\right)$ for every $j$. Moreover $\left\{\nabla w_{j}\right\}$ and $\left\{\nabla u_{j}\right\}$ generate the same Young measure and $\left\{\left|\nabla u_{j}\right|^{p(x)}\right\}$ is also equi-integrable. Since $v_{j} \in \mathscr{A}$ and $\mathscr{A}$ is weakly closed, we can get $u \in \mathscr{A}$. Moreover $u_{j} \in \mathscr{A}$. Since $v_{j}$ is a minimizing sequence for (56), we can get, by Lemma 16 ,

$$
\begin{aligned}
& \int_{\Omega \times \Omega} \int_{\mathbb{M}^{m \times N} \times \mathbb{M}^{m \times N}} W\left(x, y, \lambda_{1}, \lambda_{2}\right) d \mu_{x}\left(\lambda_{1}\right) d \mu_{y}\left(\lambda_{2}\right) d x d y \\
& \quad \leq \lim _{j \rightarrow \infty} J\left(v_{j}\right) \\
& \quad \leq \lim _{j \rightarrow \infty} J\left(u_{j}\right) \\
& \quad=\int_{\Omega \times \Omega} \int_{\mathbb{M}^{m \times N} \times \mathbb{M}^{m \times N}} W\left(x, y, \lambda_{1}, \lambda_{2}\right) d \mu_{x}\left(\lambda_{1}\right) d \mu_{y}\left(\lambda_{2}\right) d x d y .
\end{aligned}
$$

Then we can conclude that $\left\{u_{j}\right\}$ is also a minimizing sequence to $(56)$.

Step $2(m=\bar{m})$. From Step 1 , we know that $v \in \overline{\mathscr{A}}$. And

$$
\begin{aligned}
m & =\lim _{j \rightarrow \infty} J\left(u_{j}\right) \\
& =\lim _{j \rightarrow \infty} \int_{\Omega \times \Omega} W\left(x, y, \nabla u_{j}(x), \nabla u_{j}(y)\right) d x d y \\
& =\int_{\Omega \times \Omega} \int_{\mathbb{M}^{m \times N} \times \mathbb{M}^{m \times N}} W\left(x, y, \lambda_{1}, \lambda_{2}\right) d v_{x}\left(\lambda_{1}\right) d v_{y}\left(\lambda_{2}\right) d x d y \\
& \geq \bar{m} .
\end{aligned}
$$

On the other hand, for any $\mu \in \overline{\mathscr{A}}$, there exists a bounded sequence $\left\{u_{j}\right\}$ in $W^{1, p(x)}\left(\Omega, \mathbb{R}^{m}\right)$ such that $u_{j} \in \mathscr{A}$ for every $j$. We can assume that $u_{j} \rightarrow u$ and $\left\{\left|\nabla u_{j}\right|^{p(x)}\right\}$ is equi-integrable. Thus

$$
\begin{aligned}
m & \leq \lim _{j \rightarrow \infty} J\left(u_{j}\right) \\
& =\int_{\Omega \times \Omega} \int_{\mathbb{M}^{m \times N} \times \mathbb{M}^{m \times N}} W\left(x, y, \lambda_{1}, \lambda_{2}\right) d \mu_{x}\left(\lambda_{1}\right) d \mu_{y}\left(\lambda_{2}\right) d x d y .
\end{aligned}
$$

The arbitrariness of $\mu$ implies that $m \leq \bar{m}$.

Step 3. There exists $v$ such that

$$
\bar{J}(\nu)=\inf \bar{J}(\mu) .
$$

If $u_{j}$ is a minimizing sequence for (56), we can assume that $\left\{\left|\nabla u_{j}\right|^{p(x)}\right\}$ is equi-integrable and the associated Young measure $v \in \mathscr{A}$. Hence

$$
\begin{aligned}
\bar{m} & =m=\lim _{j \rightarrow \infty} J\left(u_{j}\right) \\
& =\int_{\Omega \times \Omega} \int_{\mathbb{M}^{m \times N} \times \mathbb{M}^{m \times N}} W\left(x, y, \lambda_{1}, \lambda_{2}\right) d v_{x}\left(\lambda_{1}\right) d v_{y}\left(\lambda_{2}\right) d x d y .
\end{aligned}
$$

Consequently, $v$ is a minimizer of (57).

\section{Conflict of Interests}

The authors declare that there is no conflict of interests regarding the publication of this paper.

\section{Acknowledgment}

This paper is supported by NSFC-11371110.

\section{References}

[1] O. Kováčik and J. Rákosník, "On spaces $L^{p(x)}$ and $W^{k, p(x), "}$ Czechoslovak Mathematical Journal, vol. 41, no. 116, pp. 592-618, 1991.

[2] D. E. Edmunds, J. Lang, and A. Nekvinda, "On $L^{p(x)}$ norms," Proceedings of the Royal Society of London A. Mathematical, Physical and Engineering Sciences, vol. 455, no. 1981, pp. 219-225, 1999.

[3] D. E. Edmunds and J. Rákosník, "Sobolev embeddings with variable exponent," Studia Mathematica, vol. 143, no. 3, pp. 267293, 2000.

[4] X. Fan, J. Shen, and D. Zhao, "Sobolev embedding theorems for spaces $W^{k, p(x)}(\Omega)$," Journal of Mathematical Analysis and Applications, vol. 262, no. 2, pp. 749-760, 2001.

[5] X. Fan and D. Zhao, "On the spaces $L^{p(x)}(\Omega)$ and $W^{m, p(x)}(\Omega)$," Journal of Mathematical Analysis and Applications, vol. 263, no. 2, pp. 424-446, 2001.

[6] L. Diening, P. Harjulehto, P. Hästö, and M. Růžička, Lebesgue and Sobolev Spaces with Variable Exponents, vol. 2017 of Lecture Notes in Mathematics, Springer, Berlin, Germany, 2011.

[7] J. Chabrowski and Y. Fu, "Existence of solutions for $p(x)$ Laplacian problems on a bounded domain," Journal of Mathematical Analysis and Applications, vol. 306, no. 2, pp. 604618, 2005, Corrigendum: Journal of Mathematical Analysis and Applications, vol. 323, p. 1483, 2006.

[8] X. Fan, Q. Zhang, and D. Zhao, "Eigenvalues of $p(x)$-Laplacian Dirichlet problem," Journal of Mathematical Analysis and Applications, vol. 302, no. 2, pp. 306-317, 2005.

[9] M. Galewski, "A new variational method for the $p(x)$-Laplacian equation," Bulletin of the Australian Mathematical Society, vol. 72, no. 1, pp. 53-65, 2005.

[10] E. Acerbi and G. Mingione, "Regularity results for stationary electro-rheological fluids," Archive for Rational Mechanics and Analysis, vol. 164, no. 3, pp. 213-259, 2002.

[11] E. Acerbi, G. Mingione, and G. A. Seregin, "Regularity results for parabolic systems related to a class of non-Newtonian fluids," Annales de l'Institut Henri Poincaré. Analyse Non Linéaire, vol. 21, no. 1, pp. 25-60, 2004. 
[12] P. Pedregal, "Nonlocal variational principles," Nonlinear Analysis: Theory, Methods \& Applications A: Theory and Methods, vol. 29, no. 12, pp. 1379-1392, 1997.

[13] D. Brandon and R. C. Rogers, "The coercivity paradox and nonlocal ferromagnetism," Continuum Mechanics and Thermodynamics, vol. 4, no. 1, pp. 1-21, 1992.

[14] D. Brandon and R. C. Rogers, "Nonlocal regularization of L. C. Young's tacking problem," Applied Mathematics and Optimization, vol. 25, no. 3, pp. 287-301, 1992.

[15] Z. P. Bažant and M. Jirásek, "Nonlocal integral formulations of plasticity and damage: survey of progress," Journal of Engineering Mechanics, vol. 128, no. 11, pp. 1119-1149, 2002.

[16] A. A. Pisano and P. Fuschi, "Closed form solution for a nonlocal elastic bar in tension," International Journal of Solids and Structures, vol. 40, no. 1, pp. 13-23, 2003.

[17] D. G. B. Edelen and N. Laws, "On the thermodynamics of systems with nonlocality," Archive for Rational Mechanics and Analysis, vol. 43, pp. 24-35, 1971.

[18] G. Alberti and G. Bellettini, "A nonlocal anisotropic model for phase transitions, part I: the optimal profile problem," Mathematische Annalen, vol. 310, no. 3, pp. 527-560, 1998.

[19] M. Chipot, W. Gangbo, and B. Kawohl, "On some nonlocal variational problems," Analysis and Applications, vol. 4, no. 4, pp. 345-356, 2006.

[20] P. Pedregal, Parametrized Measures and Variational Principles, Birkhäuser, 1997.

[21] L. C. Evans, Weak Convergence Methods for Nonlinear Partial Differential Equations, vol. 74 of CBMS Regional Conference Series in Mathematics, American Mathematical Society, Providence, RI, USA, 1990.

[22] G. Dolzmann, N. Hungerbühler, and S. Müller, "Non-linear elliptic systems with measure-valued right hand side," Mathematische Zeitschrift, vol. 226, no. 4, pp. 545-574, 1997.

[23] J. M. Ball, "A version of the fundamental theorem for Young measures," in Partial Differential Equations and Continuum Models of Phase Transitions: Proceedings of an NSF-CNRS Joint Seminar, vol. 344, pp. 207-215, Springer, Berlin, Germany, 1989.

[24] N. Hungerbuhler, "A refinement of Ball's Theorem on Young measures," The New York Journal of Mathematics, vol. 3, pp. 4853, 1997.

[25] Y. Fu and M. Yang, "Existence of solutions for quasilinear elliptic systems in divergence form with variable growth," Journal of Inequalities and Applications, vol. 2014, article 23, 2014. 


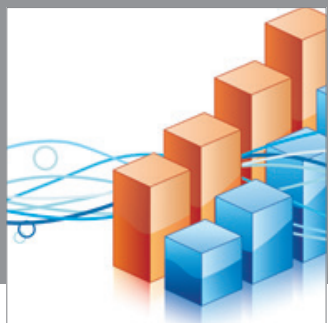

Advances in

Operations Research

mansans

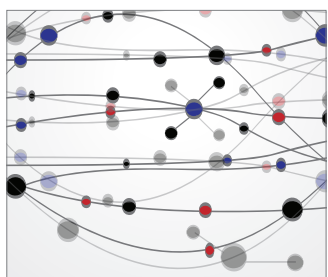

The Scientific World Journal
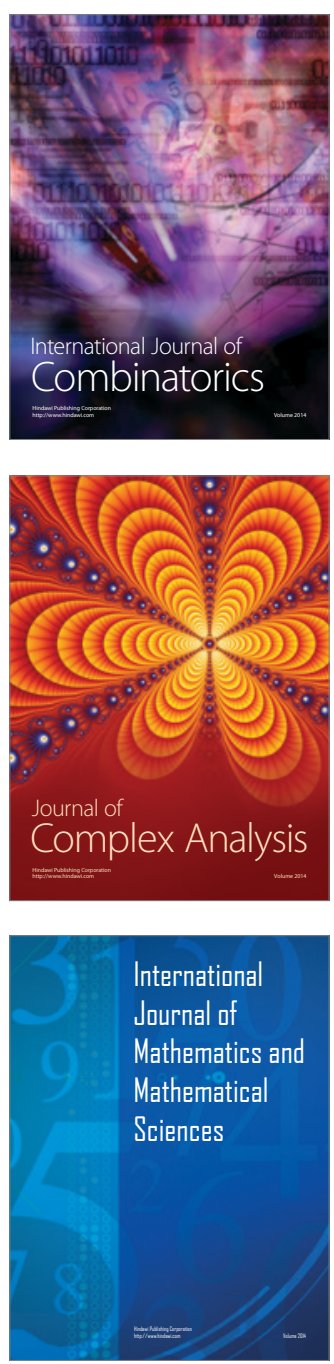
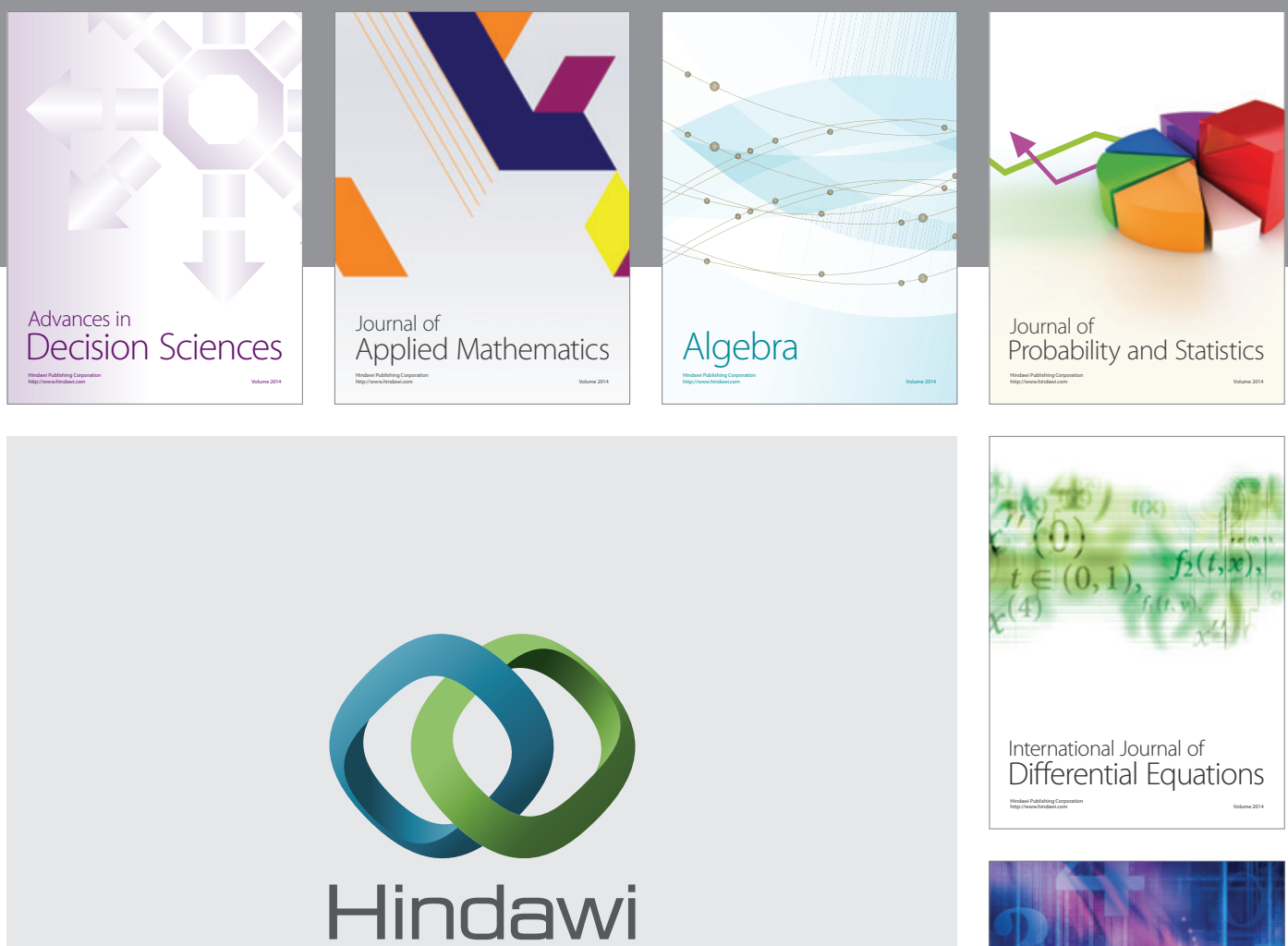

Submit your manuscripts at http://www.hindawi.com
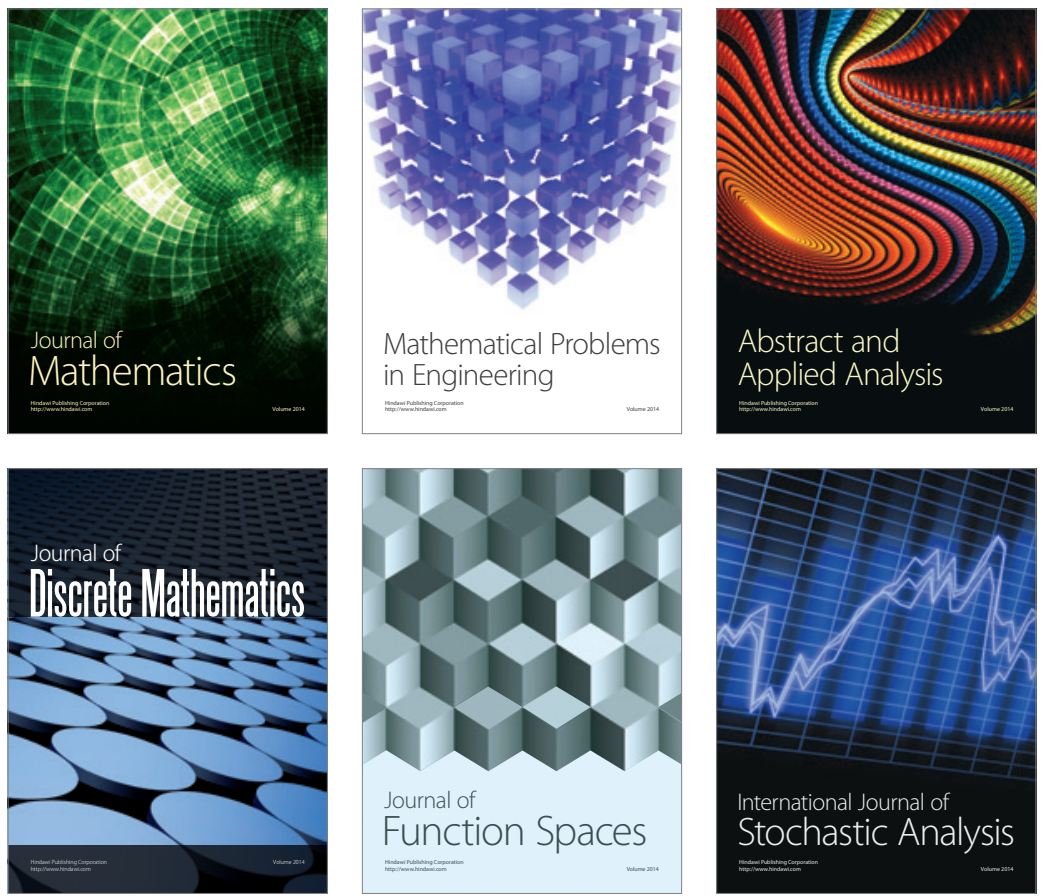

Journal of

Function Spaces

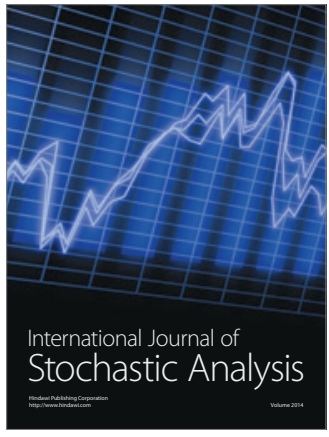

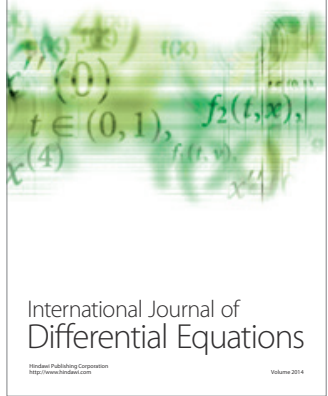
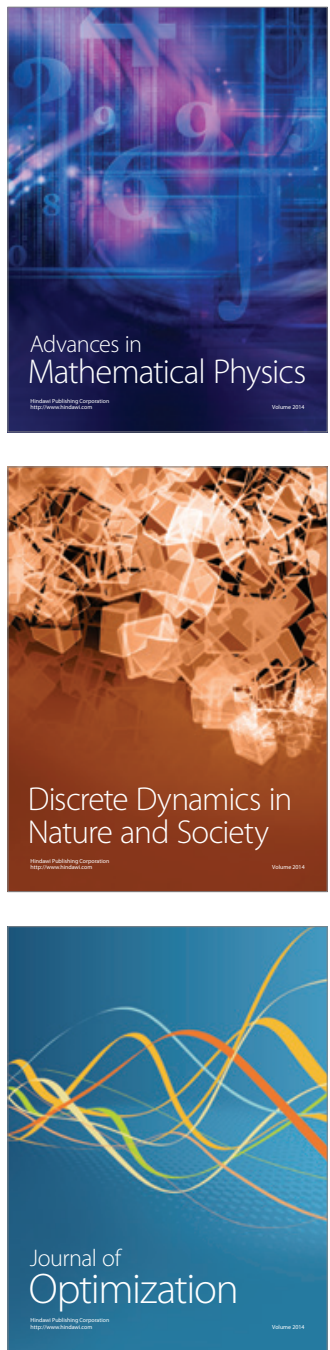